\title{
Effects of Denervation on Acetylcholine Receptor Clusters on Frog Cardiac Ganglion Neurons as Revealed by Quantitative Laser Scanning Confocal Microscopy
}

\author{
Hadley L. Wilson Horch and Peter B. Sargent \\ Departments of Stomatology and Physiology and the Neuroscience Graduate Program, University of California, \\ San Francisco, California 94143
}

\begin{abstract}
We examined the effects of denervation on clusters of nicotinic acetylcholine receptors (AChRs) on autonomic neurons in the frog heart using immunofluorescence techniques and laser scanning confocal microscopy. We showed previously that normally innervated neurons have both large, brightly stained AChR clusters and small, dim AChR clusters. A majority (80\%) of the large/bright AChR clusters are located at synaptic sites, whereas the small/dim clusters are distributed widely over the cell surface. Here, we use image analysis to identify these two classes of clusters on images generated from stacks of optical sections through neuronal cell bodies and to examine the effects of denervation on their number, size, and brightness (pixel intensity). Denervation reduces the number of large/ bright AChR clusters per cell to $<10 \%$ of sham-operated values and increases the number of small/dim clusters per cell
\end{abstract}

by two- to threefold. These changes occur at $4 \mathrm{~d}$ of denervation, the earliest time examined, and are sustained for 6 weeks. The size of large/bright AChR clusters is decreased compared with sham-operated controls, and their brightness is unchanged. The size of small/dim AChR clusters is unchanged by denervation, but their brightness is increased. Denervation results in a shift in the contribution of each AChR cluster class to the total measurable AChR pool-from one dominated by large/bright clusters to one dominated by small/dim clusters. These results show that the nerve terminals on cardiac ganglion neurons appear to exert a continual and reversible influence on the organization of the postsynaptic membrane.

Key words: acetylcholine receptors; nicotinic receptors; parasympathetic ganglia; extrasynaptic; denervation; cardiac ganglion
Innervation has a profound effect on the expression and distribution of AChRs in skeletal muscle: factors released from the nerve terminal both increase the level of transcription of AChR subunit mRNAs by synaptic nuclei and promote aggregation of AChR oligomers immediately beneath active zones (for review, see Hall and Sanes, 1993). Interruption of synaptogenesis during its early stages, either in vivo (Slater, 1982) or in vitro (Kuromi and Kidokoro, 1984), reverses the process of differentiation and leads to the loss of synaptic AChR clusters. Curiously, denervation of adult skeletal muscle does not eliminate synaptic AChR clusters (Frank et al., 1975; Porter and Barnard, 1975). This is presumably because of the survival of nerve-released factors in the synaptic basal lamina, such as agrin (for review, see Nastuk and Fallon, 1993) and neuregulin/acetylcholine receptor-inducing activity (Jo et al., 1995; Sandrock et al., 1995), and to long-lasting changes in the cytoskeleton within the muscle fiber (for review, see Froehner, 1993; Apel and Merlie, 1995).

In contrast to what is known about regulation of $\mathrm{ACh}$ expression and distribution in skeletal muscle, our understanding of the

Received Nov. 9, 1995; revised Dec. 13, 1995; accepted Dec. 14, 1995.

This work was supported by a Grant-in-Aid from the American Heart Association, California affiliate, and by National Institutes of Health Grants NS24207 and RR07131. We thank the following people: Dr. Jon M. Lindstrom (University of Pennsylvania) and Dr. Steven Carlson (University of Washington) for antibodies; Drs. Rita Balice-Gordon, Michele Jacob, Chien-Ping Ko, Patricia Steen, and Wes Thompson for reviewing a draft of this manuscript; and Dr. Jeff Toy for animal care.

Correspondence should be addressed to Dr. Peter B. Sargent, Division of Oral Biology, HSW-604, University of California, San Francisco, CA 94143-0512.

Ms. Wilson Horch's present address: Department of Neurobiology, Duke University Medical Center, Durham, NC 27710.

Copyright (C) 1996 Society for Neuroscience $0270-6474 / 96 / 161720-10 \$ 05.00 / 0$ process in neurons is primitive, even in autonomic ganglia, where the regulation of neuronal AChRs has been studied extensively (for review, see Lukas and Bencherif, 1992; Sargent, 1993; McGehee and Role, 1995). There is general agreement that innervation stimulates expression of $\mathrm{AChRs}$ and promotes an increase in $\mathrm{ACh}$ sensitivity (for review, see McGehee and Role, 1995). In the chicken ciliary ganglion, early surgical removal of the preganglionic neurons delays or reduces the expression of AChRs (Engisch and Fischbach, 1992; Arenella et al., 1993; Schwartz Levey et al., 1995). When previously innervated ciliary ganglion neurons are denervated, they appear to maintain synaptic clusters of surface AChRs for some time (Jacob and Berg, 1988). This suggests that innervation in the ciliary ganglion may have long-lived effects on $\mathrm{AChR}$ clusters, as in muscle. In the frog cardiac ganglion, however, we found that $\Lambda \mathrm{ChR}$ clusters do not survive long periods of denervation. Thus, AChR clusters, visualized with electron microscope (EM) immunoperoxidase techniques, are smaller and more dispersed on the neuronal surface $2-3$ weeks after denervation than on normally innervated cells (Sargent and Pang, 1988). This might be explained if denervation results in the fragmentation of existing AChR clusters or in their replacement by a population of clusters expressed uniquely by denervated neurons.

The advent of laser scanning confocal microscopy (LSCM) has permitted us to examine, at submicron resolution, the distribution of AChRs on the surface of frog cardiac ganglion neurons that have been reconstructed from optical sections. We have expanded our previous EM study to address three questions. (1) Is a denervation-induced reorganization of $\mathrm{AChR}$ clusters evident when LSCM is used to view cluster organization directly at the level of whole, reconstructed neurons? (2) How rapidly do 
denervation-induced changes in AChR clusters occur? (3) Is the reduction in $A C h R$ cluster size previously seen at 2-3 weeks of denervation an intermediate stage in a process whereby clusters disappear altogether?

These results have been presented previously in abstract form (Wilson and Sargent, 1994).

\section{MATERIALS AND METHODS}

Animals. Rana pipiens were obtained from Hazen (Alburg, VT) and housed at room temperature in chambers where they had access to water and a dry platform. Cardiac ganglia were dencrvatcd bilatcrally for 3-43 $\mathrm{d}$ by deeply anesthetizing animals in $4 \mathrm{~mm}$ tricaine methanesulfonate and by cutting both vagosympathetic nerves at the level of the tympanic membrane, as described by Kuffler et al. (1971). To prevent reinnervation, we sutured the proximal stump of each nerve into the skin, which denervates the heart for at least 6 months (Sargent and Dennis, 1981). Denervated and sham-operated frogs were fed twice weekly with homogenized calves' liver. Surgeries were staggered so that all tissue for an experiment was immunolabeled at one time and with the same protocol. Frogs were decapitated, double pithed, and the interatrial septum was removed and dissected in frog Ringer's (in $\mathrm{mm}$ ): $114 \mathrm{NaCl}, 2.0 \mathrm{KCl}, 2.0$ $\mathrm{CaCl}_{2}$, and 5.0 HEPES, pH 7.4.

Materials. Rat monoclonal antibodies made against electric organ AChRs (mAbs 22 and 12; Tzartos and Lindstrom, 1980; Tzartos et al., 1981) were provided by Dr. Jon M. Lindstrom (Univcrsity of Pennsylvania). A mouse monoclonal antibody against the synaptic vesicle protein SV2 (mAb 10h; Buckley and Kelly, 1985), provided by Dr. Steven Carlson (University of Washington), was used to mark synaptic boutons. Cyanine 3.18 (Cy3) and cyanine 5.18 (Cy5) conjugates of goat anti-rat and goat anti-mouse IgGs, adsorbed against conspecific IgGs, were purchased from Jackson Immunoresearch (West Grove, PA). All other reagents were purchased from Sigma (St. Louis, MO) unless otherwise indicated.

Immunofluorescence. To facilitate access of mAbs to the surface of the cardiac ganglion cells, we incubated interatrial septa at $37^{\circ} \mathrm{C}$ with $1 \mathrm{mg} / \mathrm{ml}$ collagenase (type II) for $1 \mathrm{hr}$ and then with $0.1 \mathrm{mg} / \mathrm{ml}$ protease (subtilisin, Boehringer Mannheim, Indianapolis, IN) for $0.5 \mathrm{hr}$ (Sargent and Pang, 1989). Septa were then fixed in $1 \%$ formaldehyde in $0.09 \mathrm{M} \mathrm{Na}$ phosphate buffer, $\mathrm{pH} 7.2$, for $1 \mathrm{hr}$. Double-label immunofluorescence experiments to visualize both AChRs and synaptic sites wcre done by incubating septa in the following solutions (all incubations were done at room temperature): (1) Ringer's containing $2 \%$ normal goat serum (RG) for $10 \mathrm{~min}$; (2) $5 \mathrm{nM}$ rat $\mathrm{mAb} 22$ or $\mathrm{I} 2$ (control) in RG for $1 \mathrm{hr}$; (3) RG for 30 min ( 3 changes); (4) $20 \mathrm{~nm}$ Cy3-goat anti-rat IgG in RG for $1 \mathrm{hr}$; (5) RG for $30 \mathrm{~min}$ (3 changes); (6) Ringer's containing $1 \%$ Triton X-100 for $15 \mathrm{~min}$; (7) RG for 20 min; (8) 1:200 dilution of mouse SV-2 for $1 \mathrm{hr}$; (9) RG for $30 \mathrm{~min}$ (3 changes); (10) $30 \mathrm{nM}$ Cy5-goat anti-mouse IgG for $1 \mathrm{hr}$; (11) RG for 20 $\min$ (2 changes); (12) Ringer's for $10 \mathrm{~min} ;(13) 70 \%$ glycerol $/ 30 \%$ PBS for $5 \mathrm{~min}$; and (14) $90 \%$ glycerol $/ 10 \%$ PBS for $5 \mathrm{~min}$. The anti-SV2 $\mathrm{mAb}$ labeling was done to ensure that denervation had indeed taken place (immunocytochemically demonstrable boutons disappear at $-3 \mathrm{~d}$ of denervation). Tissue was mounted in $4 \% n$-propyl gallate in $90 \%$ glycerol/ $10 \%$ PBS to retard photobleaching (Giloh and Sedat, 1982).

LSCM. Images were collected with a Bio-Rad MRC 600 laser scanning confocal microscope (Hemel Hempstead, Hertfordshire, UK) equipped with a $15 \mathrm{~mW}$ krypton/argon laser (Ion Laser Technology, Salt Lake City, UT) and filter blocks optimally designed for simultaneous detection of Cy3 and Cy5 (Sargent, 1994). All images were collected with a Nikon $60 \times$ 1.4 NA planapochromat lens (Nikon Instruments, Melville, NY) with a scan rate of $7.6 \mu \mathrm{sec}$ per $(0.1 \mu \mathrm{m})^{2}$ pixel. Photomultiplier tube amplifier gain and black levels were adjusted on an initial sample of cells so that a few pixels in the field $(\ll 1 \%)$, or anywhere in a stack of fields (for reconstructions), registered both 0 and 255 , with the remaining pixels having intermediate values. These parameters were then kept constant while imaging all neurons labeled at one time. Laser intensity was attenuated to 3 or $1 \%$ using neutral-density filters. Reconstructions were done with the confocal detector aperture at $3 \mathrm{~mm}$, yielding a measured optical section depth of $1.2 \mu \mathrm{m}$ (half-width) and a $z$ increment of $1.0 \mu \mathrm{m}$, which results in the collection of nearly all information present in the volume. Some images were collected using signal averaging $(n=2)$. Images were enhanced for display purposes (Figs. 1-3) by applying a positive $\gamma$ correction. Quantitative image analysis (see below) was performed on unenhanced images.

Image analysis. We used the OPTIMAS image analysis program (Optimas, Edmonds, WA) to analyze immunofluorescently visualized $\mathrm{AChR}$ clusters on $x y$ projections of information contained within stacks of optical sections taken at $1.0 \mu \mathrm{m}$ intervals through neuronal cell bodies. $x y$ projections were generated by displaying, at each pixel address, the brightest pixel from all the individual images in the stack (maximum projection method). Compressing information onto the $x y$ plane results in the loss or distortion of some information; for example, two clusters that occupy the same pixcl addrcsses on both the upper and lowcr halves of a neuron will appear as one cluster in $x y$ projection, and two clusters that occupy nearly identical addresses will appear as a larger cluster. Also, objects near the lateral margin of the cell body will be smaller in projection than they actually are on the neuronal surface and will have a distorted shape. The loss and/or distortion of information was judged acceptable for the purposes of this paper, because we use the results of the analysis only for comparative purposes. Thus, we examine different classes of AChR clusters that occur on the surface of sham-operated neurons and compare the properties of $\mathrm{AChR}$ clusters on denervated neurons with their properties on sham-operated neurons.

AChR clusters on sham-operated neurons (Fig. 1) fall into (at least) two categories: large, brightly stained clusters and small, dimly stained clusters. We designed a procedure using OPTIMAS to quantify the number and properties of $\mathrm{AChR}$ clusters falling into the large/bright category and the small/dim category and to follow changes in the categories after denervation. To identify objects in the $x y$ projection as AChR clusters, we defined a minimum gray value threshold (typically $20-25$ on an 8-bit scale), chosen so that no objects were identified in tissue incubated with control $\mathrm{mAb} \mathrm{12}$, and a minimum area threshold, chosen to eliminate single- and double-pixel objects (which often represent noise). This collection paradigm produced a wide distribution of objects when plotted as (log) area versus gray value (Fig. $2 A$ ). The dense array of data points in the lower left quadrant of Figure $2 A$ represents small/dim AChR clusters. To define these objects as small/dim clusters, we applied an upper area threshold and an upper gray value threshold to the data set to exclude large/bright clusters. When we examined the objects excluded by this procedure, which have sizes as large as $10 \mu \mathrm{m}^{2}$ (Fig. 2A), we found that they often corresponded not to individual large/bright AChR clusters, but to closely spaced arrays of large/bright clusters. For example, the group of $\mathrm{AChR}$ clusters at the upper left margin of the cell displayed in Figure $1 B I$ might be identified as a single AChR cluster rather than an array of 4-5 clusters. To capture these objects as individual clusters, we therefore reanalyzed the $x y$ projections by setting the gray value threshold sufficiently high to separate, at least partially, the large/bright AChR clusters from one another (typical thresholds of 60-80 on an 8-bit scale). We again set a lower area threshold to eliminate objects 1-2 pixels in size. Individual objects collected using this second analysis of the data set are not strictly comparable to objects collected in the first pass using the lower gray value threshold, because they are smaller and have a larger average gray value. An analogy is to imagine two topographical maps of a mountain range, one showing elevations $>1000$ feet and the other showing elevations $>5000$ feet. The objects in the second map are smaller than those in the first and have a greater average elevation. In summary, small/dim and large/bright AChR clusters were defined by applying two image analysis algorithms to the $x y$ projections, one with a low gray value threshold to identify small/dim clusters, and one with a higher gray value threshold to identify large/bright clusters.

In Results, we show that the two-pass procedure described in the preceding paragraph appropriately identifies most AChR clusters recognized by eye as either large/bright or small/dim. We assume that this procedure identifies populations of "large/bright clusters" and "small/dim clusters," but we have no assurance that the procedure completely separates the two populations, nor that either one of the object sets so identified is homogeneous. Thus, when we refer to "large/bright AChR clusters" and "small/dim AChR clusters," we are referring to "clusters identified as large/bright" and to "clusters identified as small/dim" by image analysis and not necessarily to nonoverlapping, homogeneous populations of objects.

Neurons were selected for reconstruction and analysis if they were sufficiently isolated from neighbors to avoid confusion as to the source of the signal. We did not image neurons immunofluorescently before choosing them for analysis. The very largest neurons in the ganglion were not imaged because they exceeded the size of the scan box $(38.4 \mu \mathrm{m} \times 38.4$ $\mu \mathrm{m} ; 10$ pixels $/ \mu \mathrm{m})$. Most of the data were gathered in three experiments, each of which entailed analyzing $180-240 \mathrm{mAb} 22$-stained neurons (15 neurons from each of 2 sham-operated and two denervated ganglia for each of 3-4 time points) as well as $30-40 \mathrm{mAb} 12$-stained neurons (negative control). A total of 770 neurons containing nearly 15,000 


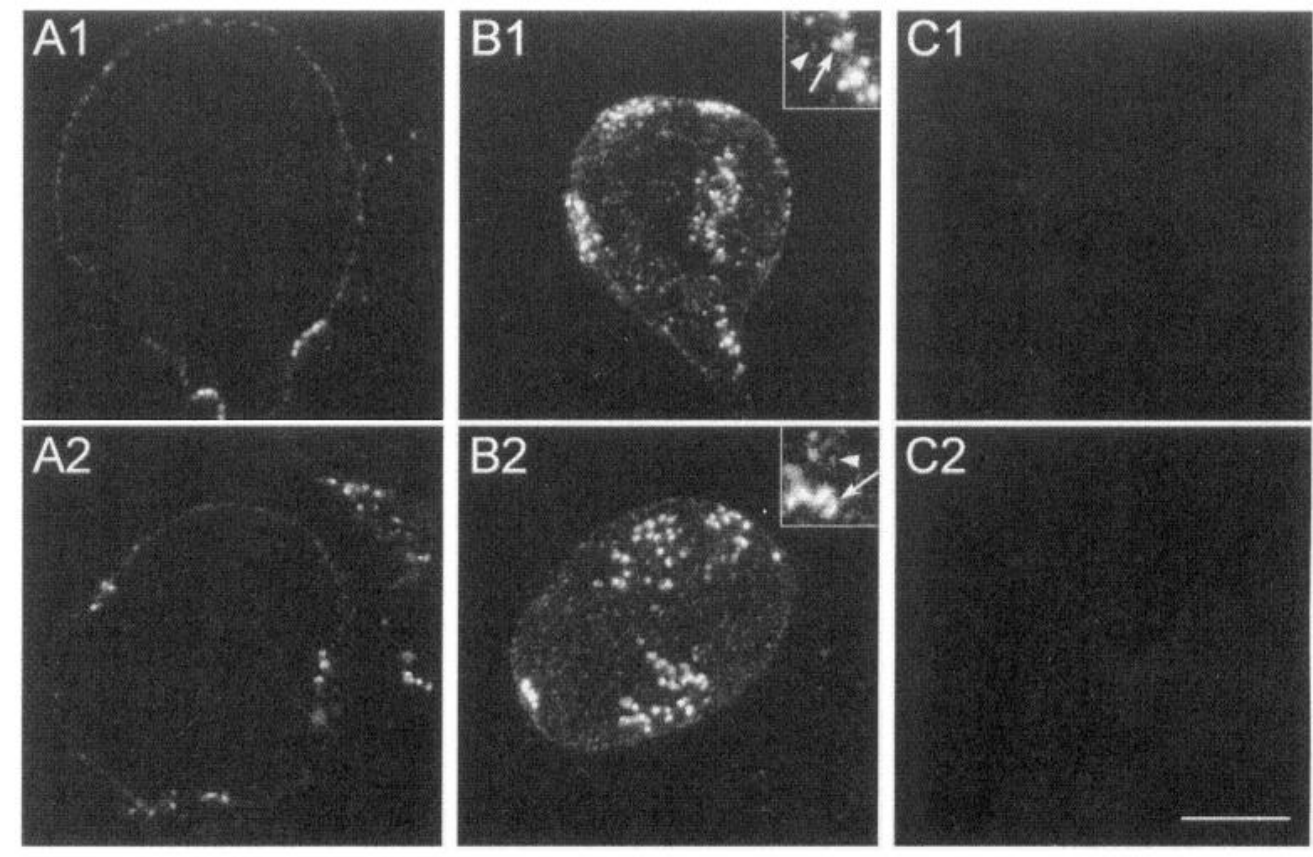

Figure 1. Confocal images illustrate that AChRs on cardiac ganglion cells occur in two classes: large/bright and small/dim. $A 1$ and $A 2$ are examples of optical sections taken through the middle of individual neuronal cell bodies from a cardiac ganglion stained with anti-AChR mAb 22 . Virtually all immunoreactivity originates from the surface of the cell. $B 1$ and $B 2$, taken from different cells than $A$, are examples of stacks of sections (maximum projection method), showing all $\mathrm{mAb} 22$ stained AChR clusters on the neuronal cell body. Insets in $B 1$ and $B 2$ display $2 \times$ magnified portions of the corresponding figures, showing large/bright AChR clusters (arrows) and small/dim AChR clusters (arrowheads). $\mathrm{Cl}$ and $\mathrm{C} 2$ are examples of stacks taken from neurons stained with control $\mathrm{mAb} 12$ using the same collection parameters as in $B 1$ and $B 2$ (same gain, black level, etc.). $D$ and $E$ show examples of stereopairs of mAb 22-stained neurons (generated by displaying two pixel-shifted stacks side-by-side). Specific staining occurs in the form of a small number of brightly fluorescent spots and a large number of small, weakly fluorescent spots, which are presumed to represent two populations of AChR clusters. The large/ bright clusters are organized into arrays, whereas the small/dim ones are widely distributed over the cell surface. The entire image was enhanced before printing by applying a $\gamma$ function of $>1$. Scale bar in $C 2$ applies to $A-C$ and is $10 \mu \mathrm{m}$; scale bar in $D$ also applies to $E$ and is $10 \mu \mathrm{m}$.
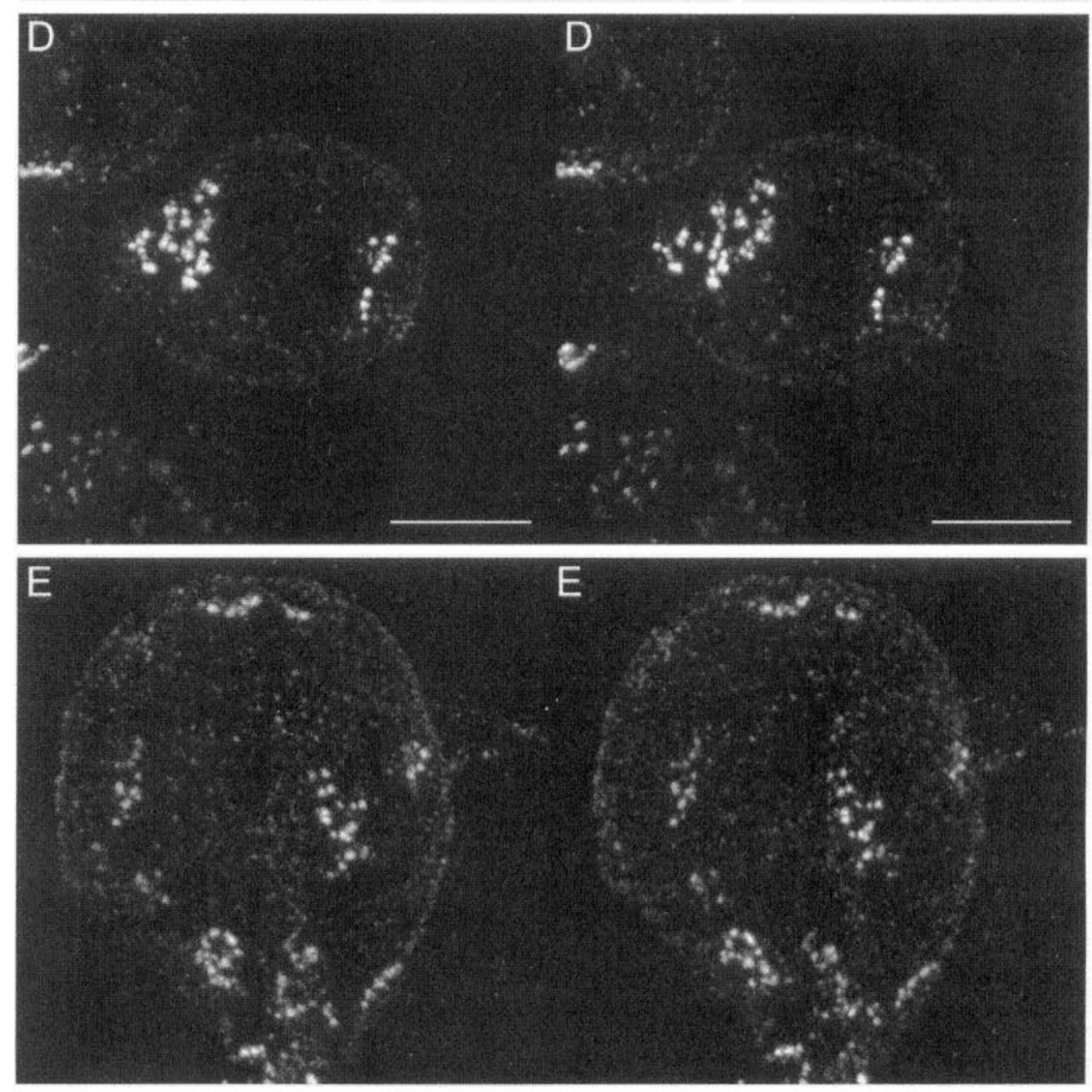

identified $\mathrm{AChR}$ clusters was imaged and analyzed. For each experiment, we collected images from all neurons with the same parameters (neutral density filter, gain, black level, zoom, etc.) and defined large/bright and small/dim AChR clusters using the same two thresholding criteria. Sample means were reasonably consistent from experiment to experiment: for example, the average area of large/bright clusters on sham-operated neurons was $0.31,0.27$, and $0.24 \mu \mathrm{m}^{2}$, whereas the average gray value for large/bright clusters on the same neurons, corrected for threshold, was 46,41 , and 49 on a scale of $0-255(n=90,120$, and 120 neurons, respectively). No obvious difference was noted for neurons denervated 

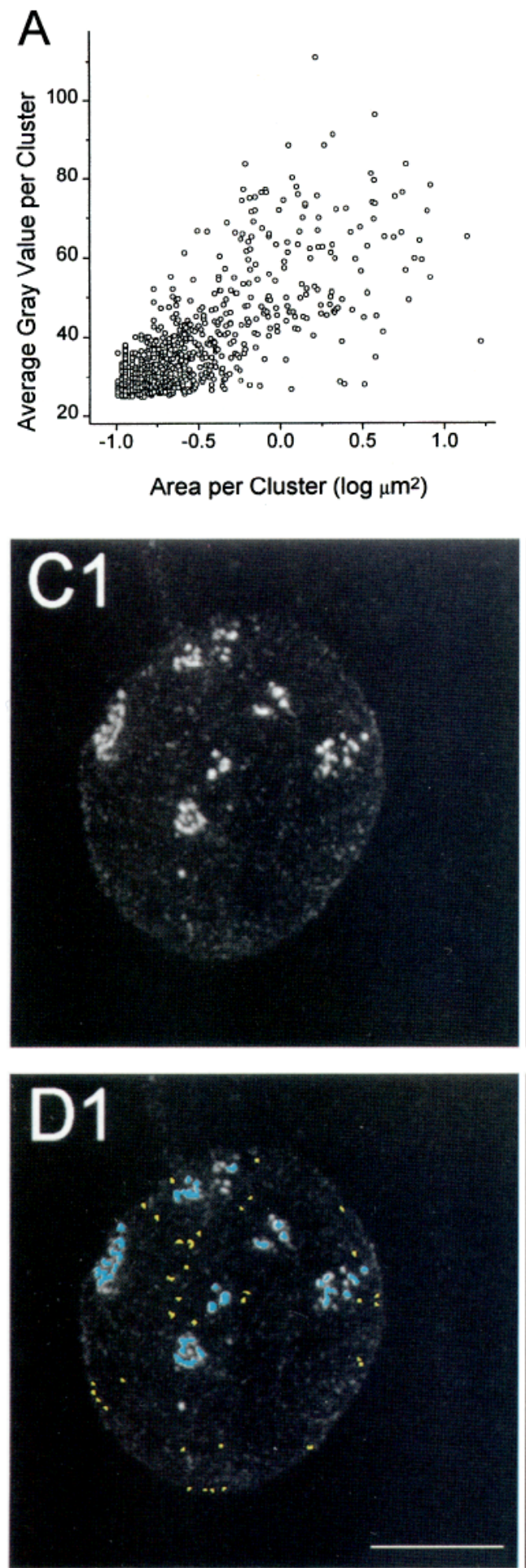
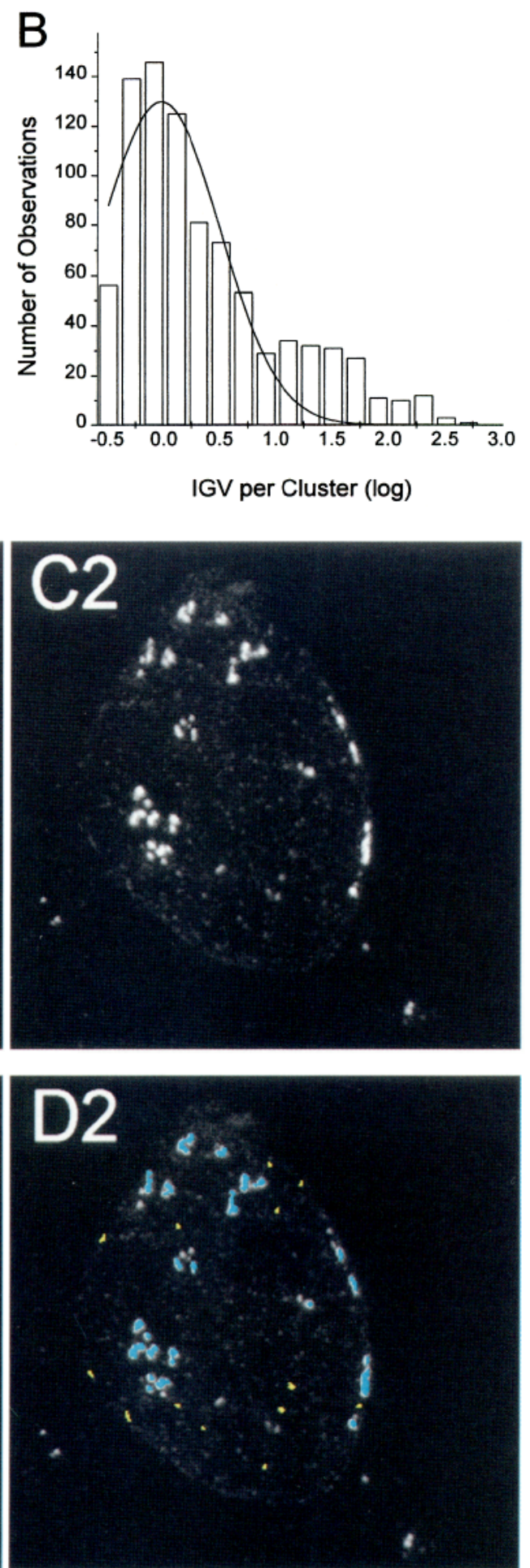

Figure 2. Quantitative image analysis indicates that $\mathrm{AChR}$ clusters on the surface of cardiac ganglion cells are diverse. $A$ displays both the area (projected onto the $x y$ plane) and the average gray value of 863 clusters from 30 sham-operated neurons. Most of the clusters are small $\left(<0.3 \mu \mathrm{m}^{2}\right)$ and dimly fluorescent (average gray value of 25-40), and a few are many times brighter and larger than these. $B$ shows a frequency histogram of these same 863 clusters, each of which was quantified by multiplying the area by the average gray value (background corrected) to yield the IGV. The distribution of IGVs, even when plotted on a log scale, is skewed to the right and cannot be fit by a single Gaussian (solid line). We used thresholding to define separate populations of objects corresponding to large/bright AChR clusters and small/ $\operatorname{dim}$ AChR clusters (described in Materials and Methods). $C$ and $D$ show the results of this thresholding protocol on two cells ( 1 and 2). $C$ shows $x y$ projections of two cells before object identification, and $D$ shows the projections after object identification. Objects identified as large/bright AChR clusters are colored cyan in $D$, and objects identified as small/dim AChR clusters are colored yellow in $D$. Not all clusters identified by eye were scored using this procedure, because thresholds for the two populations were noncontiguous and because the lower gray area threshold for small/ dim objects was not brought down to threshold (which would have resulted in our detecting some objects from control images). Note that some of the large/ bright clusters arrays (cyan) are not completely separated by this procedure. Clusters not scored as either large/ bright or small/dim appear white in $D$. Scale bar (shown in D1), $10 \mu \mathrm{m}$. for 3 , 4 , or $5 \mathrm{~d}$ and, thus, the $3-5 \mathrm{~d}$ data were pooled (" $4 \mathrm{~d}$ "), as were results for neurons denervated for 9-11 d ("10 d"), 18-21 d ("20 d"), and $40-43 \mathrm{~d}$ ("42 d").

Data collected from denervated and from sham-operated animals were compared using the Mann-Whitney test. We deemed a change in a parameter to be statistically meaningful if denervation produced a significant effect $(p<0.05)$ in all three experiments or in two of the three experiments, provided that in the third experiment there was not a significant effect in the opposite direction. We have marked those instances in which we observed a statistically significant effect, as defined here, with an asterisk in Figures $4 C$ and $5 A-D$. We used this system because the more intuitively obvious means of comparison, in which we relate the sample of three ratios (denervated mean divided by sham-operated mean) to unity, failed to indicate that denervation had an effect even in instances in which it had indeed produced a statistically significant effect in each of three experiments. As an example, the mean brightness (measured as gray value) per small/dim AChR cluster was increased at $20 \mathrm{~d}$ of denervation by 25,12 , and $84 \%$ in three experiments compared with sham-operated controls $(p<0.05$ for each comparison), yet the one-sample Student's $t$ test reveals that the mean of the values $1.25,1.12$, and 1.84 is not significantly different from unity (1). 


\section{RESULTS}

We visualized AChRs on the surface of cardiac ganglion neurons with rat antielectric organ AChR mAb 22 (Tzartos et al., 1981). We showed previously that mAb 22 binds selectively and specifically to an epitope in the postsynaptic membrane of cardiac ganglion neurons (Sargent and Pang, 1989). We assume here that it is specific for neuronal nicotinic AChRs in the ganglion.

mAb 22-labeled cardiac ganglion neurons from sham-operated ganglia show a nonuniform pattern of AChR immunoreactivity over much of their surface when displayed as optical sections (Fig. $1 A$ ), as $x y$ projections of stacks of optical sections (Fig. $1 B$ ), or as stereopairs (Fig. 1D,E). The appearance and distribution of $\mathrm{AChR}$ clusters on sham-operated neurons is similar to that on normally innervated neurons (Wilson Horch and Sargent, 1996). Replacing mAb 22 with a control mAb produced no detectable staining (Fig. 1C). Because we made no special attempt to amplify the signals collected by LSCM or to lower background, we assume that individual spots of fluorescence represent AChR clusters and not individual $\Lambda \mathrm{ChR}$ molecules. Inspection of the array of $A \mathrm{ChR}$ clusters on reconstructed neurons (Fig. $1 B, D, E$ ) suggests that they fall into two types: large, brightly fluorescent clusters (see arrows in Fig. $1 B$ insets) and smaller, less intensely stained clusters (see arrowheads in Fig. $1 B$, insets). We assume that both classes of clusters are neuronal in origin. As illustrated in the next paragraph, we have used image analysis techniques to identify these populations of clusters and examine quantitatively the effects of denervation on their number and properties.

Figure $2 A$ shows the consequence of subjecting $x y$ projections of 30 sham-operated neurons to an object collection procedure designed to identify AChR clusters (see Materials and Methods): the area of each of $863 \mathrm{AChR}$ clusters in the $x y$ projection is plotted on the $x$-axis against the average gray value (pixel intensity) on the $y$-axis. There is a high density of points in the lower left quadrant of the plot (in fact, there are many more than can be seen, attributable to stacking of points) and relatively sparse "spray" of points toward larger values of both area and average gray value. The dense population of points in the lower left quadrant of the graph represent the small/dim $\Lambda$ ChR clusters. The points with large areas and gray values actually represent closely spaced arrays of clusters rather than individual ones (see Materials and Methods). To analyze whether the objects identificd by the image analysis program can be described by a single Gaussian, we replotted the data as a frequency histogram by representing both the area and the average gray value of each AChR cluster as its integrated gray value (IGV), which is the product of the two parameters. The resultant histogram (Fig. 2B) is skewed to the right, even with the IGV per cluster binned on a log scale. Curve fitting of this histogram with Gaussians shows that the distribution cannot be well fit by a single Gaussian (Fig. 2B, solid line), and that a better fit can be achieved with two or more Gaussians (data not shown). For the purposes of this analysis, we have made the simplifying assumption that the population of AChR clusters on the neuronal surface can be described quantitatively by the sum of only two components, which are represented by the large/bright $\mathrm{AChR}$ clusters and the small/dim AChR clusters. As described in Materials and Methods, we used two separate rounds of object detection with different thresholds to identify these two populations. The ability of this image analysis scheme to identify AChR clusters that would be recognized by eye as being either large/bright or small/dim is illustrated in Figure 2, $C$ and $D$. Figure $2 C$ shows $x y$ projections of two sham-operated neurons ( $C 1$ and $C 2$ ), and Figure $2 D$ shows the same two projections, with the clusters identified as large/bright-colored cyan and the clusters identified as small/dim-colored yellow (D1 and D2). Most, but not all, of the clusters that might be identified by eye as large/bright and as small/dim are appropriately recognized by the image analysis procedure. The actual number of small/dim clusters is likely to be considerably more than the number identified by the collection scheme, because many small/dim AChR clusters are only marginally brighter than background, as can be seen by comparing Figure $1, B$ (mAb 22, experimental) and $C$ (mAb 12, control). The number of large/bright clusters is also underestimated, because clumping of individual large/bright clusters within arrays still occurs (see Fig. $2 D$ ). The underestimation of the number of cluster populations present on the neuronal surface is tolerable, because we use the analysis for comparative purposes only.

Figure 3 shows that denervation has a pronounced effect on the appearance of $\mathrm{AChR}$ clusters on the ganglion cell surface, as illustrated in $x y$ projections. Denervated neurons appear, by eye, to have lost many of their large/bright clusters and to have gained a new population of smaller clusters that are, on average, brighter than the small clusters seen on sham-operated neurons. These changes are apparent by $4 \mathrm{~d}$ of denervation, at which time transmission has failed in the ganglion (Dennis and Sargent, 1978) and synaptic boutons can no longer be demonstrated immunocytochemically using the anti-SV2 mAb $10 \mathrm{~h}$ (not shown). In the next section, we demonstrate that these denervation-induced changes are evident when neurons are analyzed without previous selection using quantitative image analysis techniques.

To gain a quantitative sense of the time course and extent of the denervation-jnduced changes in AChR clusters on the cell surface, we made systematic use of image analysis and of the protocol described in Materials and Methods to examine the effects of denervation on both the large/bright and the small/dim $\mathrm{AChR}$ clusters. At each of four times after denervation, we compared denervated neurons and sham-operated neurons with regard to the following: (1) number of clusters per cell; (2) cluster size (in $x y$ projection); (3) average gray value per cluster; and (4) IGV per cluster (the product of area and average gray value). The IGV per cluster should be a relative measure of the number of immunofluorescently detectable AChRs per cluster; by totaling these on individual neurons, we can make inferences about the contribution of both large/bright and small/dim AChR clusters to the total complement of detectable AChRs on the cell surface.

Image analysis reveals that denervation profoundly changes the number of large/bright and small/dim AChR clusters. When thresholding appropriate for the two cluster types on shamoperated neurons is used to define clusters as large/bright or small/dim, denervation is found to reduce significantly the number of large/bright clusters while increasing significantly the number of small/dim clusters. This is depicted as cumulative probability distributions in Figure $4 A, B$, for a comparison between $20 \mathrm{~d}$ sham-operated and $20 \mathrm{~d}$ denervated neurons. Denervation clearly reduces the number of $\mathrm{AChR}$ clusters identified as large/bright (Fig. $4 A$ ) while at the same time increasing the number of $\mathrm{AChR}$ clusters identified as small/dim (Fig. $4 B$ ). By performing this comparison at different times after surgery, we find that denervation's effects are evident at $4 \mathrm{~d}$ after surgery (Fig. $4 C$ ) and that they are sustained for at least 6 weeks, making it unlikely that the change in cluster properties is caused by a transient effect of denervation such as might result from the degeneration of nerve terminals (Lømo and Westgaard, 1975; Cangiano et al., 1984). 


\section{sham-operated}
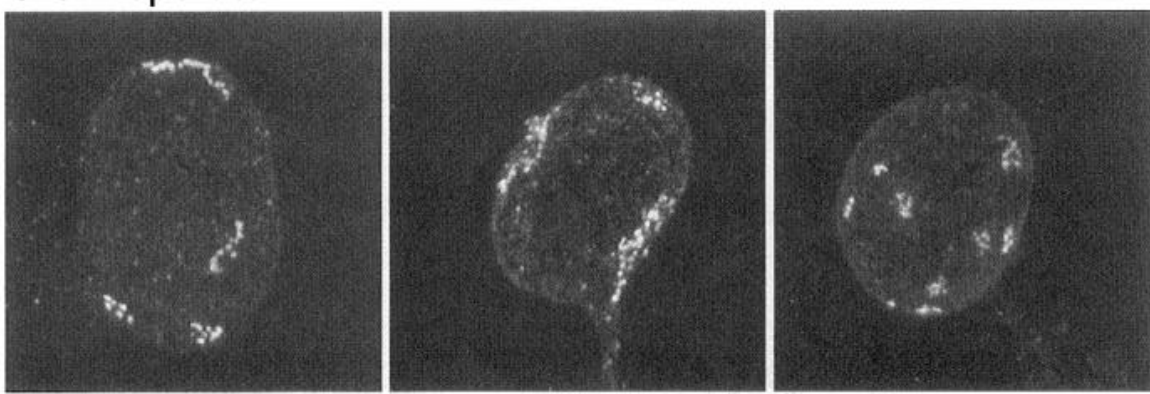

\section{4 day denervated}
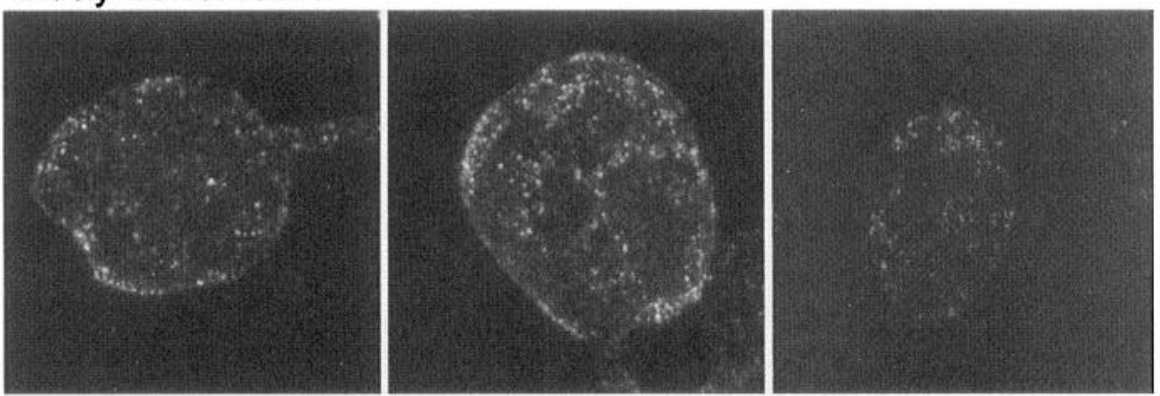

\section{0 day denervated}
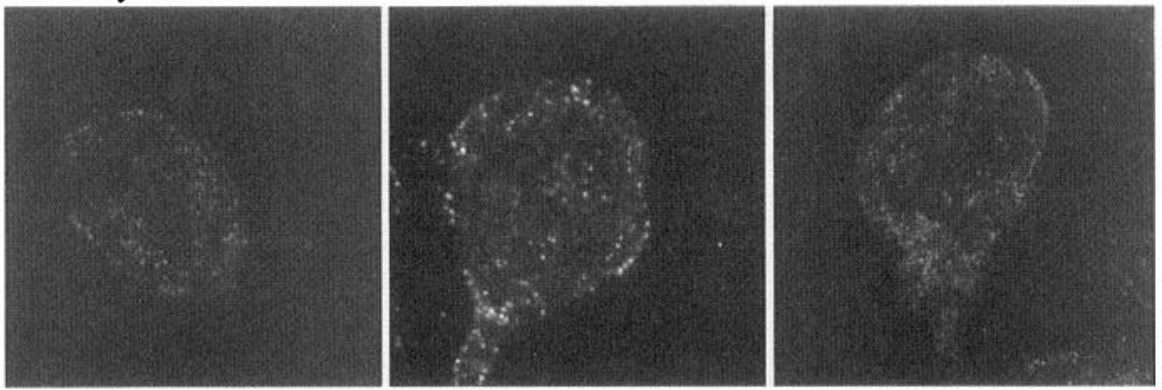

\section{0 day denervated}
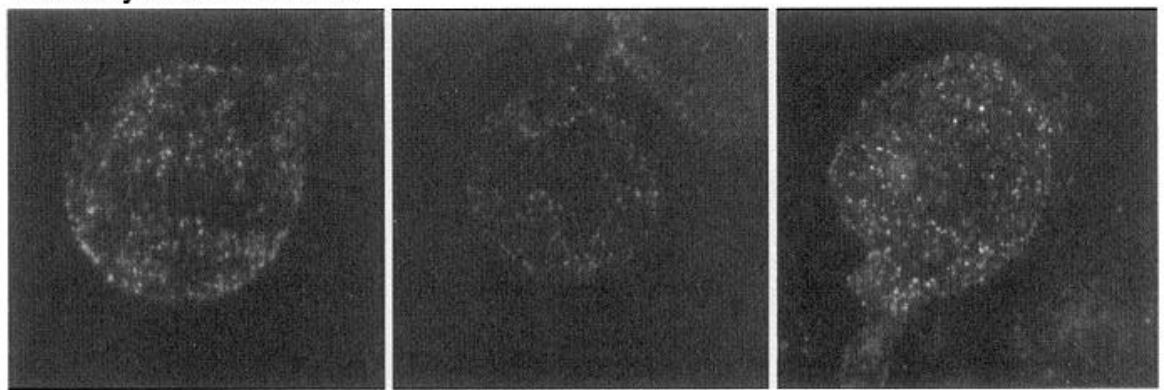

\section{2 day denervated}

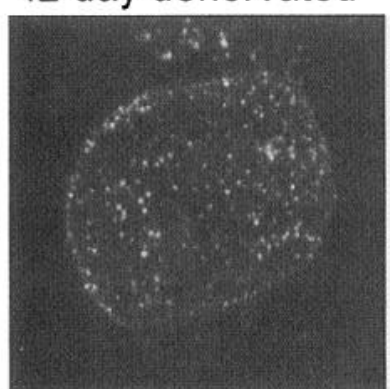

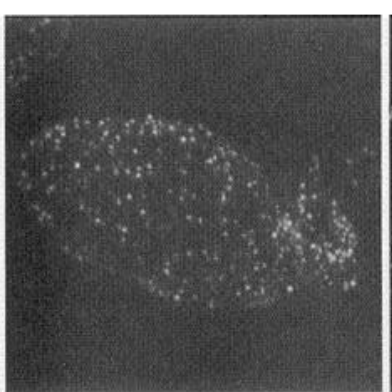

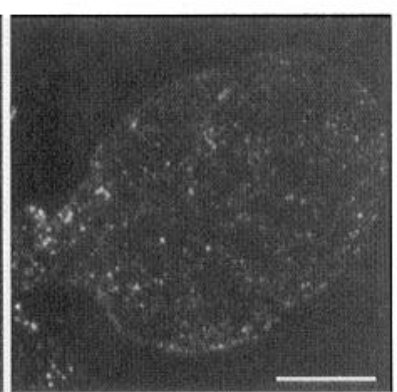

Figure 3. Denervation of cardiac ganglion neurons alters the appearance and distribution of AChR clusters on the neuronal surface. Three examples of neurons are shown for sham-operated neurons as well as for neurons denervated for 4,10 , 20 , and $42 \mathrm{~d}$. Each image is an $x y$ projection generated from a stack of optical sections (maximum projection method). Within $4 \mathrm{~d}$ of denervation, the brightest clusters are less intensely stained than those in sham-operated ganglia, and their distribution is more widespread over the neuronal surface. These changes are sustained over 6 weeks. For any one experiment, identical parameters were used to collect information from sham-operated and denervated neurons. All images were enhanced before printing by applying a $\gamma$ correction of $>1$, which reduces the perceived difference in intensity between weakly stained and strongly stained $\mathrm{AChR}$ clusters. Quantitative analyses of AChR clusters (Figs. 4, 5) were made on unenhanced images. Scale bar (shown in the bottom right image), $10 \mu \mathrm{m}$. 

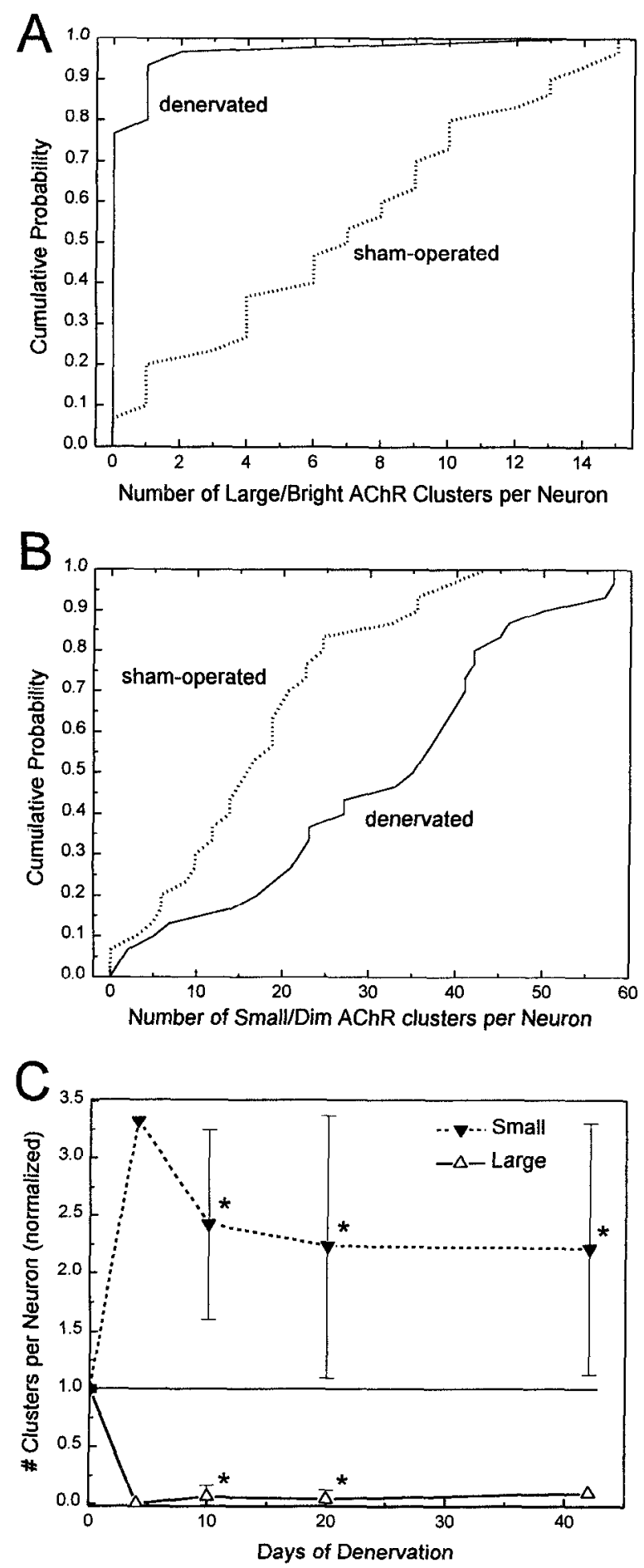

Figure 4. Effects of denervation on the number of large/bright and small/ $\operatorname{dim}$ AChR clusters. Large/bright and small/dim AChR clusters were defined using quantitative image analysis and thresholding, as described in Materials and Methods. $A$ shows a cumulative probability distribution of the number of large/bright clusters on 30 neurons denervated for $20 \mathrm{~d}$ and on 30 neurons sham-operated for $20 \mathrm{~d}$. A cumulative probability distribution, which is the integral of the frequency distribution, illustrates on the $y$-axis the fraction of neurons having fewer clusters than the corresponding value on the $x$-axis. $B$ shows a cumulative probability distribution of the number of small/dim clusters on 30 neurons denenvated for $20 \mathrm{~d}$ and on 30 neurons sham-operated for $20 \mathrm{~d}$. $C$ shows the time-resolved effects of denervation on both large/bright $(\triangle)$ and small/dim ( $\mathbf{v})$ AChR clusters. Each point represents the mean of two $(4 \mathrm{~d})$ experiments or the mean $\pm \mathrm{SD}$ of three experiments; in each experiment, the average number of clusters per cell for 30 denervated neurons was
Denervation produced little or no change in the average gray value of large/bright AChR clusters and only moderate changes in the average gray value of small/dim AChR clusters. A summary plot of the results of three experiments shows that large/bright AChR cluster gray values were not significantly changed by denervation except at $10 \mathrm{~d}$, when they were reduccd by --20\% (Fig. $5 A$ ). Small/dim AChR clusters had significantly increased average gray values at 20 and $42 \mathrm{~d}$ of denervation, but not at earlier times (Fig. 5A). Thus, these changes are modest and slow to develop compared with the change in the number of clusters in each category.

Denervation produced a small but significant change in the size of surviving large/bright AChR clusters. The size of small/dim AChR clusters was changed little, if at all. The summary plot indicates that the reduction in size of surviving large/bright clusters is statistically significant by $10 \mathrm{~d}$ of denervation, after which it is maintained at $\sim 80 \%$ of normal (Fig. $5 B$ ).

The IGV per cluster, the product of average gray value and cluster size, should be a relative measure of the number of $\mathrm{AChRs}$ per cluster. Because denervation produced a moderate decrease in the average gray value of surviving large/bright AChR clusters and a moderate decrease in the size of these clusters, it follows that the IGV per large/bright cluster should also decrease, and indeed it does. The summary plot shows that IGV per cluster declines after denervation in relation to sham-operated controls (Fig. 5C). This reduction is modest, statistically significant at $10 \mathrm{~d}$ of denervation, and sustained for at least 6 weeks after surgery. By contrast, denervation produced a moderate increase in the IGV per cluster for small/dim AChR clusters (Fig. 5C).

All of the denervation-induced changes noted here serve to decrease the fraction of detectable AChRs on the surface that arise from clusters identified as large/bright and to produce concomitant increases in the contribution of clusters identified as small/dim. If we plot the sham-normalized fraction of AChRs accounted for by large/bright clusters, we find, as expected, that it declines precipitously within $4 \mathrm{~d}$ of denervation and remains low for at least 6 weeks (Fig. 5D). The fraction of all AChRs accounted for by the small/dim AChR clusters increases markedly with the same time course. If we were to assume that the IGV per cluster is directly proportional to AChR number (which is unlikely to be strictly true), we can calculate that large/bright AChR clusters account for $\sim 80 \%$ of all detectable AChR clusters on the neuronal surface of sham-operated neurons and $5 \%$ in denervated neurons. Conversely, the contribution from small/dim AChR clusters increases from $20 \%$ of the total in sham-operated animals to $95 \%$ of the total in denervated neurons.

When we sum the contributions to IGV from both the large/ bright and the small/dim clusters on individual neurons, we arrive at a relative measure of the total complement of detectable AChRs on the surface (total IGV). Total IGV does not change significantly after denervation (data not shown); this is because the loss in signal attributable to large/bright clusters is accounted for by a corresponding increase in signal from the small/dim

divided by the average number for their sham-operated counterparts. Asterisks denote instances in which denervation had a significant cffect on the number of clusters per cell, as defined in Materials and Methods. The large/bright cluster data for one $42 \mathrm{~d}$ experiment were not used to generate the displayed value because the sham-operated mean was $<10 \%$ of that observed in the other two experiments (1.0 vs 11.0 and 11.5$). C$ shows that denervation nearly eliminates AChR clusters defined as large/ bright and increases the number of clusters defined as small/dim by twoto threefold. 

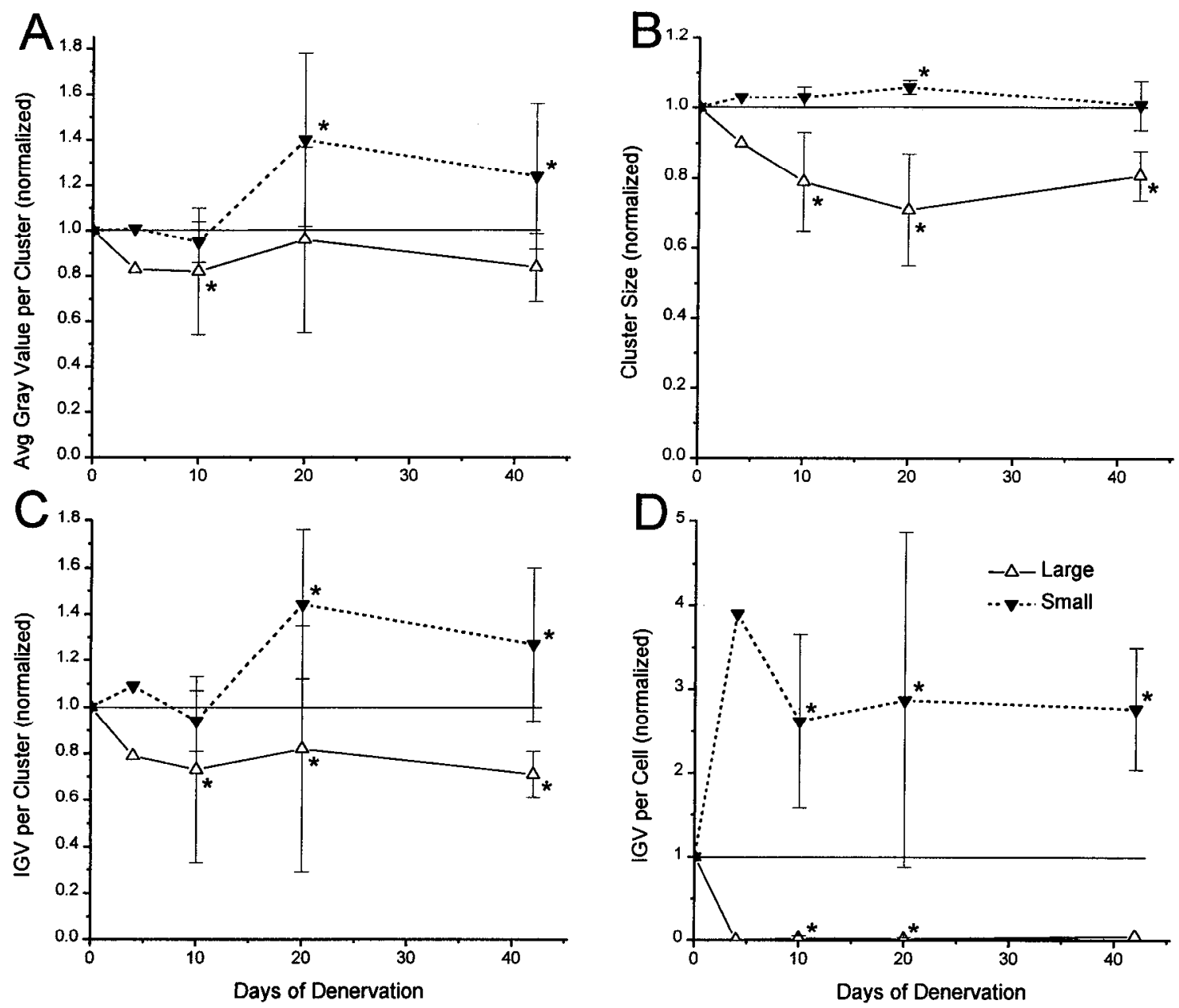

Figure 5. Effects of denervation on the average gray value (pixel intensity) $(A)$, size $(B)$, IGV per cluster $(C)$, and IGV per cell $(D)$ for AChR clusters defined as large/bright $(\triangle)$ and as small/dim $(\nabla)$. Each point represents the mean of two $(4 \mathrm{~d})$ or the mean \pm SD of three experiments; in each experiment, the mean of the value derived from 30 denervated neurons was normalized by dividing it by the value derived from 30 sham-operated and age-matched neurons. The large/bright cluster data for one $42 \mathrm{~d}$ experiment were not used to generate the displayed value in $D$ because the sham-operated mean was $<10 \%$ of that observed in the other two experiments (1.0 vs 11.0 and 11.5). Asterisks denote instances in which denervation had a significant effect on the appropriate variable, as defined in Materials and Methods. Denervation produced no consistent effects on the size of large/bright clusters, but it increased the brightness of small/dim clusters at 20 and $42 \mathrm{~d}$ of denervation $(A)$. Denervation reduced the size of large/bright clusters but had no consistent effects on the size of small/dim clusters $(B)$. Denervation decreased the IGV per cluster for large/bright clusters and increased it for small/dim clusters $(C)$. Denervation profoundly altered the relative contribution of large/bright and small/dim clusters to the total detectable complement of AChRs on the neuronal surface $(D)$ : it decreased the contribution of large/bright clusters while increasing the contribution of small/dim clusters.

clusters. This suggests that the principal effect of denervation is not to alter the number of detectable AChRs on the neuronal surface, but rather their organization.

\section{DISCUSSION}

We previously analyzed the effects of denervation on AChR clusters visualized as immunoperoxidase-stained patches in the electron microscope (Sargent and Pang, 1988). Although the spatial resolution of this procedure is high, we were forced to make inferences about the effects of denervation without the benefit of directly observing any one neuron's entire array of AChR clusters: for example, we inferred that the distribution of AChR clusters was altered by virtue of changes in the frequency with which they were found adjacent to one another in sections through the cell body. Moreover, we inferred that denervation had changed the number of clusters per cell by relying on estimates of cluster number generated by dividing the total surface area occupied by clusters by the average cluster size (each of these parameters was itself calculated). At the time these earlier studies were performed, we were unable to image the surface complement of AChR clusters on whole cells. In the intervening time, we have gained access to a confocal microscope and have designed a novel filter set that allows us to perform imaging without exciting the autofluorescent granules that populate the cytosol of frog cardiac ganglion neurons (Sargent, 1994). This, in turn, allowed us to ask whether the inferences we made by calculation and on the basis of a relatively small sample of high-resolution micrographs (many thin sections but few neuronal equivalents) were correct. In the current study, we have been able to confirm, with good sampling, the findings we made previously (Sargent and Pang, 1988). Denervation does indeed profoundly alter the appearance and arrangement of AChR clusters, as can been seen directly in $x y$ projections of sham-operated and denervated neurons (Fig. 3). The ability to sample the entire surface of neurons rapidly has allowed us to examine the time course of a phenomenon that we 
previously studied at only one time point ( $2-3$ weeks) (Sargent and Pang, 1988).

By separately defining large/bright AChR clusters, which are largely synaptic, and small/dim AChR clusters, which are largely extrasynaptic, we sought to learn the fate of each cluster class after denervation. Although denervation produced modest reductions in the size of large/bright AChR clusters, its most pronounced effect was on the number of these clusters, which was reduced to $<10 \%$ of controls. Concomitantly, denervation markedly increased the number of clusters defined as small/dim while causing only modest changes in their brightness. Thus, denervated neurons are missing one class of objects found on normally innervated neurons while having increased numbers of another class of objects. One possible explanation, which we first suggested based on earlier work (Sargent and Pang, 1988), is that denervation leads to the fragmentation of large/bright AChR clusters ( $80 \%$ of which are located at synaptic sites) and to their dispersal. This would explain several results: (1) the reduction in the number of large/bright clusters per cell; (2) the change in surface distribution of the brightest clusters; (3) the increase in average gray value of small/dim clusters, which now includes products of the fragmentation of the original large/bright clusters; and (4) the increase in the number of small/dim clusters per cell. It is also possible that denervation does not fragment and disperse large/bright clusters, but instead leads to their complete elimination. However, this would not explain the increase in the number of small/dim clusters unless we were to propose, additionally, that denervated neurons generated extra AChR clusters that are more brightly stained than the small/dim clusters present on normally innervated neurons. A complete description of the events after denervation will necessitate further research regarding the influence of innervation on the balance between the rate of insertion of AChRs into the membrane and the rate of their removal. The loss of innervation appears to eliminate a signal needed for the assembly of large AChR clusters. Whether, in the absence of this signal, existing AChRs are dispersed or eliminated in situ will have to await experiments in which we follow existing AChR clusters by repeatedly imaging identificd cells (Balice-Gordon and Lichtman, 1993). The prospects for doing this in the cardiac ganglion are poor, but it might be possible in the mouse submandibular ganglion (Purves et al., 1987).

Does denervation eliminate all large/bright $\mathrm{AChR}$ clusters or only the $80 \%$ that are colocalized with synaptic sites (Wilson Horch and Sargent, 1996)? When all pairwise comparisons between denervated and sham-operated neurons are pooled (regardless of time of denervation), we find that the number of large/bright clusters is $5 \pm 8 \%$ of control, which is significantly different from $20 \%$ ( $p=0.002$ by Wilcoxan signed rank test). Thus, denervation eliminates even those large/bright AChR clusters that are originally extrasynaptic. An understanding of this may come from the location of these clusters; $95 \%$ of them are situated within $2 \mu \mathrm{m}$ of existing synaptic boutons (Wilson Horch and Sargent, 1996). These clusters, like their synaptic counterparts, thus may owe their integrity to the presence of intact innervation.

We previously found by EM that denervation resulted in a reduction, by a factor of three, in the total membrane area occupied by AChR clusters (Sargent and Pang, 1988); in the current study, however, we find no consistent effect of denervation in the total AChR signal (IGV per cell; data not shown). Neither of these methods is likely to provide an accurate measure of the total complement of AChRs on the cell surface. Moreover, we now know that the two procedures do not visualize the same subset of AChRs, because the small/dim AChR clusters are not visualized by the $\mathrm{EM} /$ immunoperoxidase technique (Wilson Horch and Sargent, 1996). Our LSCM results are consistent with the EM results in the sense that the EM data show a denervationinduced reduction in the size of $\mathrm{ACl}$ R clusters that populate the large/bright class. Our data are also consistent with previous work in which we examined the effects of denervation on the number of binding sites for neuronal-bungarotoxin (n-Bgt; also known as $\kappa$-bungarotoxin). The lack of change in total IGV per cell (from both classes of clusters) suggests that denervation does not change the total number of surface AChRs, a result also seen in more quantitative experiments in which the number of surface AChRs recognized by n-Bgt was assessed by autoradiography (Sargent et al., 1991). It should be pointed out that we do not know whether mAb 22 and n-Bgt recognize the same class(es) of AChRs in the cardiac ganglion (Halvorsen and Berg, 1987).

The changes in the distribution and properties of AChRs detected by LSCM in this study may have little to do with the development of supersensitivity to $\mathrm{ACh}$ in the cardiac ganglion (Kuffler et al., 1971; Roper, 1976; Dennis and Sargent, 1979). We have failed to find evidence that ACh supersensitivity is caused by a change in the total number of AChRs on the ganglion cell surface (Sargent et al., 1991). Other evidence, based on the response of ganglion cells to $\mathrm{ACh}$ versus carbamylcholine (Streichert and Sargent, 1992), suggests that supersensitivity is caused by a reduced effectiveness of acetylcholinesterase (AChE). However, we have found no evidence for reduced levels of $\mathrm{AChE}$ in the ganglion after denervation (Streichert and Sargent, 1990) (P. Sargent, H. Wilson Horch, E. Garrett, S. Matthews, unpublished results). A remaining possibility is that supersensitivity is caused by the redistribution of AChRs (or AChE) so as to reduce the effectiveness of $\mathrm{AChE}$ in hydrolyzing $\mathrm{ACh}$ that would otherwise reach AChRs. But the currently documented changes in detectable AChRs, which occur as soon as $4 \mathrm{~d}$ after denervation, precede the onset of supersensitivity, which takes 2 weeks to develop fully (Dennis and Sargent, 1979). This suggests that the redistribution of AChRs is not likely to help explain denervation supersensitivity to $\mathrm{ACh}$ in the ganglion.

The speed with which large/bright AChR clusters are lost (4 d in a poikilotherm) invites speculation about the mechanism by which intact innervation controls $\mathrm{AChR}$ distribution. One influence of terminals that is interrupted immediately by the surgery is electrical activity; it would be interesting to know whether the disruption of synaptic AChR clusters can be mimicked by application of tetrodotoxin in cuffs to the preganglionic nerves.

One striking difference between these results and those in skeletal muscle (and in the chick ciliary ganglion) (Jacob and Berg, 1988) relates to the stability of synaptic AChR clusters after denervation. Long-term denervation in adult skeletal muscle does not disrupt synaptic AChR clusters (Frank et al., 1975; Porter and Barnard, 1975) presumably because nerve-induced changes in the extracellular matrix and/or the cytoskeleton of the muscle cell are long-lasting. Why not in the frog cardiac ganglion? If terminals are not physically stable in this preparation (Purves et al., 1987), then there may be no advantage to stabilizing individual elements, such as AChR clusters, so that they can exist in the absence of their synaptic partner.

\section{REFERENCES}

Apel ED, Merlie JP (1995) Assembly of the postsynaptic apparatus. Curr Opin Neurobiol 5:62-67. 
Arenella LS, Oliva JM, Jacob MH (1993) Reduced levels of acetylcholine receptor expression in chick ciliary ganglion neurons developing in the absence of innervation. J Neurosci 13:4525-4537.

Balice-Gordon RJ, Lichtman JW (1993) In vivo observations of pre- and postsynaptic changes during the transition from multiple to single innervation at developing neuromuscular junctions. J Neurosci 13:834-855.

Buckley KM, Kelly RB (1985) Identification of a transmembrane glycoprotein specific for secretory vesicles of neural and endocrine cells. J Cell Biol 100:1284-1294.

Cangiano A, Magherini PC, Pasino E, Pellegrino M, Risaliti R (1984) Interaction of inactivity and nerve breakdown products in the origin of acute denervation changes in rat skeletal muscle. J Physiol (Lond) 355:345-365.

Dennis MJ, Sargent PB (1978) Multiple innervation of normal and reinnervated parasympathetic neurones in the frog cardiac ganglion. J Physiol (Lond) 281:63-75.

Dennis MJ, Sargent PB (1979) Loss of extrasynaptic acetylcholine sensitivity upon reinnervation of parasympathetic ganglion cells. J Physiol (Lond) 289:263-275.

Engisch KL, Fischbach GD (1992) The development of ACh- and GABAactivated currents in embryonic chick ciliary ganglion neurons in the absence of innervation in vivo. J Neurosci 12:1115-1125.

Frank E, Gautvik K, Sommerschild H (1975) Cholinergic receptors at denervated mammalian motor end-plates. Acta Physiol Scand 95:66-76.

Froehner SC (1993) Regulation of ion channel distribution at synapses. Annu Kev Neurosci 16:347-368.

Giloh H, Sedat JR (1982) Fluorescence microscopy: reduced photobleaching of rhodamine and fluorescein protein conjugates by $n$-propyl gallate. Science 217:1252-1255.

Hall ZW, Sanes JR (1993) Synaptic structure and development: the neuromuscular junction. Neuron 10:99-121.

Halvorsen SW, Berg DK (1987) Affinity labeling of neuronal acetylcholine receptor subunits with an $\alpha$-neurotoxin that blocks receptor function. J Neurosci 7:2547-2555.

Jacob MH, Berg DK (1988) The distribution of acetylcholine receptors in chick ciliary ganglion neurons following disruption of ganglionic connections. J Neurosci 8:3838-3849.

Jo AS, Zhu X, Marchionni MA, Burden SJ (1995) Neuregulins are concentrated at nerve-muscle synapses and activate ACh-receptor gene expression. Nature 373:158-161.

Kuffler SW, Dennis MJ, Harris AJ (1971) The development of chemosensitivity in extrasynaptic areas of the neuronal surface after denervation of parasympathetic ganglion cells in the heart of the frog. Proc $\mathrm{R}$ Soc Lond [Biol] 177:555-563.

Kuromi H, Kidokoro Y (1984) Denervation disperses acetylcholine receptor clusters at the neuromuscular junction in Xenopus clusters. Dev Biol 104:421-427.

Lomo T, Westgaard RH (1975) Control of $\Lambda$ Ch sensitivity in rat muscle fibers. Cold Spring Harb Symp Quant Biol 40:263-274.

Lukas RJ, Bencherif M (1992) Heterogeneity and regulation of nicotinic acetylcholine receptors. Int Rev Neurobiol 34:25-131.

McGehee DS, Role LW (1995) Physiological diversity of nicotinic acetylcholine receptors expressed by vertebrate neurons. Annu Rev Physiol 57:521-546.

Nastuk MA, Fallon JR (1993) Agrin and the molecular choreography of synapse formation. Trends Neurosci 16:72-76.
Porter CW, Barnard EA (1975) Distribution and density of cholinergic receptors at the motor end-plates of a denervated mouse muscle. Exp Neurol 48:542-556.

Purves D, Voyvodic JT, Magrassi L, Yawo H (1987) Nerve terminal remodeling visualized in living mice by repeated examination of the same neuron. Science 238:1122-1126.

Roper S (1976) The acetylcholine sensitivity of the surface membrane of multiply innervated parasympathetic ganglion cells in the mudpuppy before and after partial denervation. J Physiol (Lond) 254:455-473.

Sandrock AW, Goodearl AD, Yin QW, Chang D, Fischbach GD (1995) ARIA is concentrated in nerve terminals at neuromuscular junctions and at other synapses. J Neurosci 15:6124-6136.

Sargent PB (1993) The diversity of neuronal nicotinic acetylcholine receptors. Annu Rev Neurosci 16:403-443.

Sargent PB (1994) Double-label immunofluorescence with the laser scanning confocal microscope using cyanine dyes. NeuroImage $1: 288-295$.

Sargent PB, Dennis MJ (1981) The influence of normal innervation upon abnormal synaptic connections between frog parasympathetic neurons. Dev Biol 81:65-73.

Sargent PB, Pang DZ (1988) Denervation alters the size, number, and distribution of clusters of acetylcholine receptor-like molecules on frog cardiac ganglion neurons. Neuron 1:877-886.

Sargent PB, Pang DZ (1989) Acetylcholine receptor-like molecules are found in both synaptic and extrasynaptic clusters on the surface of neurons in the frog cardiac ganglion. J Neurosci 9:1062-1072.

Sargent PB, Bryan GK, Streichert LC, Garrett EN (1991) Denervation does not alter the number of nicotinic acetylcholine receptors on autonomic neurons in the frog cardiac ganglion. J Neurosci 11:3610-3623.

Schwartz Levey M, Brumwell CL, Dryer SE, Jacob MH (1995) Innervation and target tissue interactions differentially regulate acetylcholine receptor subunit mRNA levels in developing neurons in situ. Neuron $14: 153-162$

Slater CR (1982) Neural infuence on the postnatal changes in acetylcholine receptor distribution at nerve-muscle junctions in the mouse. Dev Biol 94:23-30.

Streichert LC, Sargent PB (1990) Differential effects of denervation on acetylcholinesterase activity in parasympathetic and sympathetic ganglia of the frog, Rana pipiens. J Neurobiol 21:938-949.

Streichert LC, Sargent PB (1992) The role of acetylcholinesterase in denervation supersensitivity in the frog cardiac ganglion. J Physiol (Lond) 445:249-260.

Tzartos SJ, Lindstrom JM (1980) Monoclonal antibodies used to probe acetylcholine receptor structure: localization of the main immunogenic region and detection of similarities between subunits. Proc Natl Acad Sci USA 77:755-759.

Tzartos SJ, Rand DE, Einarson BL, Lindstrom JM (1981) Mapping of surface structures of electrophorus acetylcholine receptor using monoclonal antibodies. $\mathrm{J}$ Biol Chem 256:8635-8645.

Wilson HL, Sargent PB (1994) Effects of denervation upon acetylcholine receptor clusters in autonomic neurons as determined by quantitative laser scanning confocal microscopy. Soc Neurosci Abstr 20:1139.

Wilson Horch HL, Sargent PB (1996) Synaptic and extrasynaptic distribution of two distinct populations of nicotinic acetylcholine receptor clusters in the frog cardiac ganglion. J Neurocytol 25:67-77. 